\title{
Space Division Multiplexing using Multi-Element Fibers
}

\author{
V. J. F. Rancaño, S. Jain, T. C. May-Smith, J. K. Sahu, P. Petropoulos and D. J. Richardson \\ Optoelectronic Research Centre, University of Southampton, Southampton, UK SO17 1BJ
}

\begin{abstract}
Multi-element Fibers (MEFs) are presented as an alternative spatial division multiplexing (SDM) technology which can address some of the drawbacks that current implementations exhibit. The benefits obtained from this fiber geometry show that MEF can be a cost-effective solution for SDM implementation in commercial optical networks.
\end{abstract}

Keywords- Optical Fiber Communications, Spatial Division Multiplexing, Multi-Element Fibers

\section{INTRODUCTION}

The present annual telecommunications traffic growth rate, which is close to $30 \%[1]$, may lead to a turning point in the trend of an ever decreasing cost-per-bit due to the large numbers of fibers and wavelength division multiplexing (WDM) systems required in order to accommodate it.

SDM has been proposed as a way of improving both the cost and power efficiency of current optical networks by grouping parallel WDM systems into SDM systems with more favorable economics. For SDM ever to be adopted commercially it must deliver similar levels of performance and reliability as existing WDM systems whilst offering clear economic benefits. In addition, the high cost of nationwide fiber deployment, especially in rural areas, and the long lifetime of transmission links dictates the need for compatibility with standard single mode fibers (SSMFs) for SDM systems to be adopted in commercial networks.

Currently, the two main approaches to SDM are based on multi-core fibers (MCFs) or mode division multiplexing (MDM) in multi-mode fibers. While impressive demonstrations have been performed in both technologies[2,3], both are ultimately constrained by crosstalk between the spatial channels. This crosstalk predominantly comes from two different sources: the inherent mode coupling between the different cores and modes, in MCF and MDM geometries respectively, and the coupling at fiber and device interconnections - especially at splice points. In comparison with WDM systems based on SSMFs, the mitigation of interchannel crosstalk is a new requirement, and multipleinput-multiple-output (MIMO) processing is used to compensate for it when the crosstalk levels are above $25 \mathrm{~dB}$ [4]. The use of MIMO however reduces cost and power efficiency, and practical implementation will become further complicated by the anticipated future application of Flexigrid spectrum allocation [5] and the subsequent increase in the number of wavelengths per system. Moreover, since MIMO requires access to all the spatial channels to successfully decouple the information channels, it constrains the flexibility of the network by restricting switching in the spatial dimensions [6]. Multi-element Fibers (MEFs) have been proposed as an alternative to these existing approaches [7-9]. This fiber geometry exhibits very low levels of crosstalk and is fully compatible with SSMF systems, making it a suitable

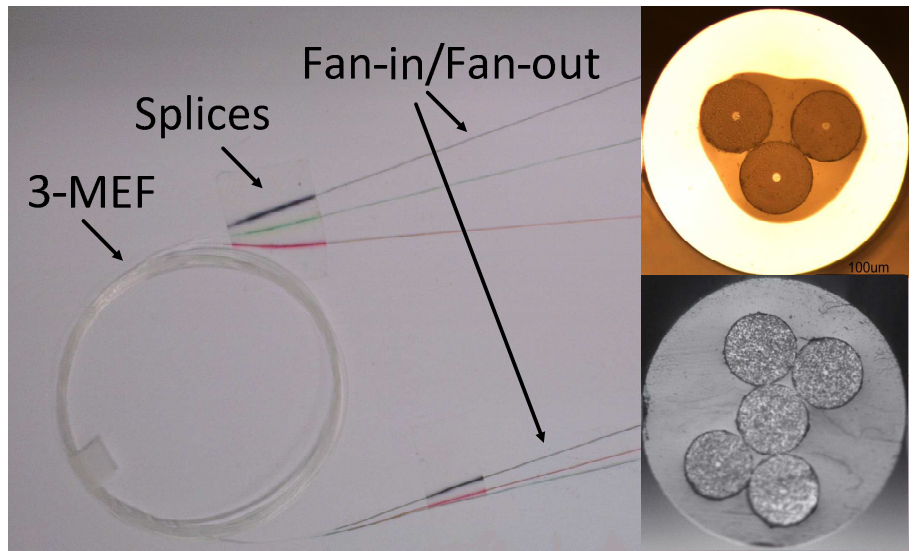

Figure 1. Photography of a 3-element MEF(left), its cross-section (top right) and the cross-section of a 5-element MEF (bottom right)

candidate for an effective implementation of SDM in commercial optical networks.

\section{MULTI-ELEMENT FIBER TECHNOLOGY}

MEF consists of a bundle of single-mode fiber-elements drawn together in a common polymer coating, each element comprising an independent core and cladding. This geometry allows each of the fiber elements to be smaller than SSMF offering the prospect of reducing the micro-bending losses relative to individual fibers of the same size. At the same time, the inline bundling process in MEF eliminates the need for a two-stage fabrication process that is required for a similar bundling of SSMF.

The fiber geometry provides easy access to the individual elements simply by removing the common coating, as displayed in Fig. 1. In this manner, each element can be spliced individually with standard splicers to provide crosstalk-free connections. In addition, the connection to standard WDM components can be performed by splicing standard single mode fiber pigtails at each end of the elements, avoiding the need for the development of custom fan-in/fan-out devices.

The distance between the fiber-elements of MEF must be accurately controlled during the manufacturing process in order to obtain the required level of robustness necessary for deployment in optical transmission systems and without introducing any additional losses. The optimum spacing for a transmission fiber is achieved when the elements are very close, but without any physical contact (see top right inset of Fig. 1)[7]. The attenuation and dispersion characteristics for MEF has been observed to be similar to that of a SSMF drawn from the same starting preform[10], showing no additional penalty with respect to SMFs. MEF exhibits crosstalk levels 
under $100 \mathrm{~dB}$ between the various elements and full compatibility with standard WDM components[10].

MEF technology lends itself directly to the implementation of SDM amplifiers. If the fiber-elements are made to touch one another, a cladding-pumped multi-element erbium doped fiber amplifier (ME-EDFA) can be manufactured[11]. This design is similar to the GT-Wave concept used in high power fiber lasers[12]. By making the central fiber-element without a core, a pump source can be coupled into the ME-EDFA and the pump light diffuses into the erbium-doped fiber-elements (see bottom right inset of Fig. 1). Since the geometry of the elements is similar to the transmission MEF, ME-EDFAs show no measurable crosstalk between elements [11]. Since a single multimode pump diode is shared among all the elements, this pumping scheme exhibits a cost efficiency that with the absence of any crosstalk between elements, this pumping scheme makes for a cost effective SDM amplifier technology.

\section{MULTI-ELEMENT FIBERS IN SPATIAL DIVISION MULTIPLEXING SYSTEMS}

The advantages of MEF technology in SDM environments were demonstrated in a recent transmission experiment[7,9]. A schematic diagram of which is shown in Fig. 2. The system comprised two sections of transmission MEF followed by a ME-EDFA amplification.

The experiment showed several key points that are important to highlight. The elements performed as independent WDM systems with no bit-error rate (BER) degradation measured down to a sensitivity level of $10^{-8}$ (independently of the number of elements in use and powers transmitted through them). No MIMO processing was necessary to recover any of the signals, owing to the crosstalk-free environment of the $\mathrm{MEF}$ and from the crosstalk-free splices.

The small attenuation differences between the distinct elements within a MEF, an effect that can occur over time in real systems caused by inhomogeneous degradation or by fiber bending during deployment, could be addressed individually for each element by placing a standard manual variable optical attenuator (MVOA) at the input of the ME-EDFA. This feature allows the commissioning and reconfiguration of this type of SDM system as isolated WDM systems, and can reduce the complexity and risks of field operations during reconfiguration tasks.

Finally, fully backward compatibility with SSMF, WDM modulators/demodulators and off-the-shelf components was shown without requiring any further development, which proves to a large degree the cost efficiency of this approach.

\section{CONCLUSIONS}

MEF technology exhibits several intrinsic features that make it a strong candidate for a flexible, crosstalk-free, robust and operation-friendly SDM solution that meets the requirements of commercial optical systems. Nevertheless, the technology is still at early stages of research, and several developments relating to the optimization of element density, increase in the number of elements and so on are still under way.

\section{ACKNOWLEDGMENTS}

This work was supported by the EPSRC grant EP/I01196X: Transforming the Future Internet: The Photonics Hyperhighway.

\section{REFERENCES}

[1] "Will Traffic Growth Break the Internet - And Can Optical Communications Help?" OFC 2014 Rump Session.

[2] J. Sakaguchi et al., "Large-capacity transmission over a 19-core fiber" OFC OW1L3 (2013)

[3] V.A.J.M. Sleiffer et al., "73.7 Tb/s (96x3x256-Gb/s) mode-divisionmultiplexed DP-16QAM transmission with inline MM-EDFA" ECOC Th.3.C.4 (2012)

[4] P. Winzer et al., "Penalties from In-Band Crosstalk for Advanced Optical Modulation Formats" ECOC Tu.5.B.7 (2011)

[5] ITU-T Recommendation G.694.1

[6] P. Winzer ECOC "Spatial Multiplexing: The next frontier in network capacity Scaling” We.1.D.1 (2013)

[7] V. J. F. Rancaño et al, "First Demonstration of an Amplified Transmission Line Based on Multi-Element Fibre Technology". ECOC PD1.C.2 (2013)

[8] J. K. Sahu et al, "Multi-Element Fiber for Space-Division Multiplexing" Proc. SPIE 9009, Next-Generation Optical Communication: Components, Sub-Systems, and Systems III, 90090C (2013)

[9] S. Jain, V. J. F. Rancaño, T. C. May-Smith, P. Petropoulos, J. K. Sahu, and D. J. Richardson, "Multi-Element Fiber for Space-division Multiplexing Operations," Opt. Express, 22 (4), 3787-3796 (2014)

[10] S. Jain et al., "Multi-Element Fibre for Space-division Multiplexed Transmission" ECOC Mo.4.A.2 (2013)

[11] S. Jain et al., "Cladding-pumped Er/Yb-doped multi-element fiber amplifier” Workshop on Specialty Optical Fibers W5.4 (2013)

[12] S.-U. Alam et al., "Low cost multi-port reconfigurable erbium doped cladding pumped fibre amplifier" ECOC Paper 5.4.3 (2000)

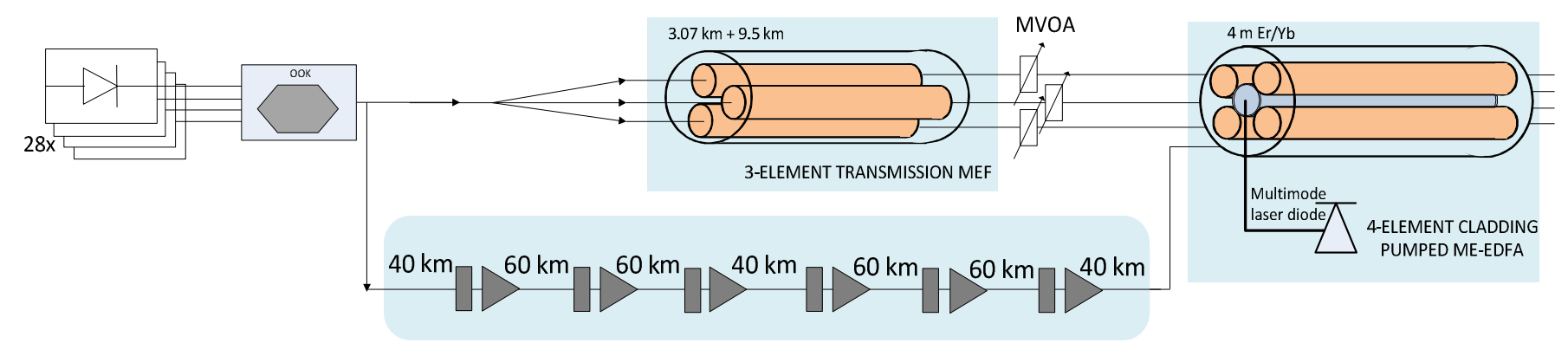

Figure 2. System diagram of the transmission experiment performed with MEF and ME-EDFA showing backward compatibility[9] 